\title{
Two new species of nematode parasites, Cucullanus epinepheli sp. n. (Cucullanidae) and Procamallanus (Spirocamallanus) sinespinis sp. n. (Camallanidae), from marine serranid and haemulid fishes off New Caledonia
}

\author{
František Moravec $^{1}$ and Jean-Lou Justine ${ }^{2}$
}

\author{
${ }^{1}$ Institute of Parasitology, Biology Centre of the Czech Academy of Sciences, České Budějovice, Czech Republic; \\ ${ }^{2}$ Institut Systématique, Évolution, Biodiversité, Muséum National d'Histoire Naturelle, Sorbonne Universités, Paris, France
}

\begin{abstract}
Based on light and scanning electron microscopical studies, two new species of parasitic nematodes are described from marine perciform fishes off New Caledonia: Cucullanus epinepheli sp. n. (Cucullanidae) from the intestine of the brownspotted grouper Epinephelus chlorostigma (Valenciennes) (Serranidae) and Procamallanus (Spirocamallanus) sinespinis sp. $\mathrm{n}$. from the intestine of the silver grunt Pomadasys argenteus (Forsskål) (Haemulidae). Cucullanus epinepheli sp. n. differs from its congeners mainly in possessing a unique structure of the anterior, elevated cloacal lip with a large posterior outgrowth covering the cloacal aperture and in the presence of cervical alae and two small preanal papillae on the median dome-shaped precloacal elevation. This is the second known nominal species of this genus parasitising fishes of the family Serranidae and the second representative of Cucullanus Müller, 1777 recorded from fishes in New Caledonian waters. Procamallanus (Spirocamallanus) sinespinis sp. n. is mainly characterised by 10-12 spiral ridges in the buccal capsule, the presence of wide caudal alae, three pairs of pedunculate preanal papillae, two unequally long spicules $(465-525 \mu \mathrm{m}$ and $218-231 \mu \mathrm{m})$ and by the tail tip with a knob-like structure in the male, and the broad, rounded tail with a terminal digit-like protrusion without cuticular spikes in the female. This is the fifth nominal species of the subgenus Spirocamallanus Olsen, 1952 reported from fishes in New Caledonian waters.
\end{abstract}

Keywords: Nematoda, helminth parasite, Seuratoidea, Camallanoidea, Epinephelus, Pomadasys, South Pacific

Despite extensive studies of parasites of marine fishes carried out by the junior author of this paper (J.-L. J.) and his collaborators in New Caledonia during two recent decades, the nematode fauna of these hosts in New Caledonian waters remains little known. This also concerns representatives of the Cucullanidae Cobbold, 1864 and Camallanidae Railliet et Henry, 1915, although members of these two families are frequent parasites of Indo-Pacific fishes, where many species have been reported.

Parasitological examinations of some marine fishes off New Caledonia conducted in 2009 and 2010 revealed, among other helminths, two species of adult cucullanid and camallanid nematodes in serranid and haemulid hosts, respectively, which proved to be new species of $\mathrm{Cucul}$ lanus Müller, 1777 and Procamallanus (Spirocamallanus) Baylis, 1923. The results of detailed studies of these parasites are presented herein. Both host species are Indo-Pacific tropical marine fishes targeted by commercial fisheries (Froese and Pauly 2016).
The only two species of Cucullanus previously recorded from off New Caledonia are C. bourdini Petter et Le Bel, 1992 and C. bulbosus (Lane, 1916), parasites of lutjanid fishes (Lutjanidae) and a carangid fish (Carangidae), respectively (Petter and Le Bel 1992, Moravec and Justine 2011a, 2016), whereas four nominal species of the subgenus Spirocamallanus have been reported in New Caledonian waters: $S$. philippinensis Machida et Taki, 1985 [= syn. of $P$. (S.) guttatusi (Andrade-Salas, Pineda-López et García-Magaña, 1994)] (probably misidentified) from Digramma pictum (Thunberg) (see Baillon 1991), P. (S.) longus Moravec, Justine et Rigby, 2006 from Zebrasoma scopas (Cuvier) (Acanthuridae), P. (S.) monotaxis (Olsen, 1952) from Lethrinus genivittatus Valenciennes and L. variegatus Valenciennes (both Lethrinidae) and $P$. (S.) variolae Moravec, Justine et Rigby, 2006 from Variola albimarginata and Epinephelus fasciatus (Forsskål) (both Serranidae) (Moravec et al. 2006, Justine 2010, Moravec and Justine 2011b).

Address for correspondence: F. Moravec, Institute of Parasitology, Biology Centre of the Czech Academy of Sciences, Branišovská 31 , 370 05 České Budějovice, Czech Republic. Phone: +420 38777 5432; Fax: +420 38 5310388; E-mail: moravec@paru.cas.cz

Zoobank number for article: urn:lsid:zoobank.org:pub:BCBD06D2-A104-47A7-8C74-21D580518F29 


\section{MATERIALS AND METHODS}

Fish were caught off Nouméa, New Caledonia, or purchased from the fishmarket in Nouméa. Fish were identified using standard morphological keys; in addition, specimen JNC3241 [Epinephelus chlorostigma (Valenciennes)] was subjected to a molecular study (Schoelinck et al. 2014) and a tissue sample was deposited in the Muséum National d'Histoire Naturelle (MNHN) collection. Parasites were collected by the wash method of Justine et al. (2012). The nematodes were fixed in hot 70\% ethanol. For light microscopical examination, they were cleared with glycerine. Drawings were made with the aid of a Zeiss drawing attachment. Specimens used for scanning electron microscopy were transferred to $4 \%$ formalin, postfixed in $1 \%$ osmium tetroxide (in phosphate buffer), dehydrated through a graded acetone series, critical-point-dried and sputter-coated with gold; they were examined using a JEOL JSM-7401F scanning electron microscope at an accelerating voltage of $4 \mathrm{kV}$ (GB low mode). All measurements are in micrometres unless otherwise indicated. The fish nomenclature and classification adopted follows FishBase (Froese and Pauly 2016).

\section{RESULTS}

Family Cucullanidae Cobbold, 1864

\section{Cucullanus epinepheli sp. n.}

Figs. 1-3

ZooBank number for species:

urn:Isid:zoobank.org:act:C16237C7-781C-4BA1-BA36-D3D5F09A6216

Description of male (three specimens; measurements of holotype in parentheses). Medium-sized nematodes. Body whitish, elongate, 7.9-9.0 mm (7.9 mm) long, maximum width 326-449 (326) at middle of body; width at level of oesophastome 218-258 (218), at middle of oesophagus 204-258 (204). Narrow lateral alae present, extending from approximately level of base of pseudobuccal capsule to level of posterior oesophagus (Figs. 1B, 2E,F); length of alae in one paratype 898, width 24. Cephalic end somewhat asymmetrical in lateral view (Figs. 1A, 2A). Oral aperture dorsoventrally elongate, surrounded by raised narrow membraneous ala (collarette) supported by row of $c a 100$ minute basal teeth. Four submedian cephalic papillae and pair of lateral amphids present (Figs. 1C, 2A-C). Oesophagus muscular, expanded at anterior end to form bulbous pseudobuccal capsule (oesophastome); posterior part of oesophagus also expanded, broader than oesophastome (Fig. 1A,B). Length of entire oesophagus $1.1-1.2 \mathrm{~mm}(1.1 \mathrm{~mm})$, representing $13-14 \%$ (14\%) of whole body length; length of oesophastome 313-354 (313), its width 190-218 (190); minimum width of oesophagus 95-109 (95); maximum width of posterior part of oesophagus 218-258 (218). Oesophagus opens into intestine through large valve (Fig. 1A,B).

Distance of nerve ring from anterior extremity 408-422 (408), representing 35-37\% (37\%) of oesophageal length. Deirids small, slightly asymmetrical, situated short distance anterior to end of oesophagus (Figs. 1A,B,D, 2D-F). Postdeirids not found. Excretory pore just posterior to oesopha- go-intestinal junction (Fig. 1A). Deirids and excretory pore 891-979 (891) and 1,197-1,360 (1,197), respectively, from anterior end of body. Posterior end of body curves ventrally. Ventral precloacal sucker and subventral oblique muscle bands in region between sucker and cloacal aperture well developed (Figs. 1F, 2G, 3A); distance of sucker from cloaca 870-884 (870). Anterior lip of cloaca elevated, forming large posterior transverse-oval outgrowth covering cloacal aperture; large median dome-shaped formation containing 2 papillae present just anterior to anterior cloacal lip (Figs. 1E-G, 2G, 3A-D); elevated anterior cloacal lip and median dome-shaped formation 15-18 (18) high and both together 63-72 (63) long in lateral view.

Spicules equal, alate, 748-789 (748) long, with pointed distal ends (Fig. 1F,H), representing 9-10\% (10\%) of body length; width of distal end of spicule in holotype (20) in lateral view. Gubernaculum small, rod-like in lateral view, well sclerotised,150-163 (163) long (Fig. 1E,F). Genital papillae 10 pairs: 4 pairs of subventral preanal papillae, 1 pair of subventral adanal papillae and 5 pairs of postanal papillae, of which 3 pairs subventral and 2 pairs dorsolateral. First pair of subventral postanal papillae just posterior to cloacal aperture, second and third pairs of subventral postanals in posterior half of tail; dorsolateral pairs of postanals at level of first and second pairs of subventrals (Figs. 1E,F,G, 2G, 3A-C). Phasmids small, lateral, slightly anterior to level of second pair of subventral postanal papillae (Figs. 1E-G, 3B). Tail conical, 204-286 (245) long, with rather obtuse tip (Figs. $1 \mathrm{E}-\mathrm{G}, 3 \mathrm{~A}-\mathrm{C}$ ).

Female: Not known.

Type host: Brownspotted grouper Epinephelus chlorostigma (Valenciennes) (Perciformes: Serranidae). The three fish specimens examined ranged between $380-570 \mathrm{~mm}$ in fork length and 719-2,700 $\mathrm{g}$ in weight. Tissue sample of fish JNC3141 deposited in the collections of the MNHN as MNHN-IC-ICTI-2145.

Site of infection: Intestine.

Type locality: Along slope off the barrier reef, off Nouméa, New Caledonia, coordinates $22^{\circ} 34^{\prime} \mathrm{S} ; 1_{166^{\circ}} 26^{\prime} \mathrm{E}$ (collected 1 December 2009).

Prevalence and intensity: 1 fish infected $/ 3$ fish examined; 3 nematodes.

Deposition of type specimens: Holotype, Muséum National d'Histoire Naturelle, Paris, MNHN JNC 3141; 1 paratype, Helminthological Collection of the Institute of Parasitology, Biology Centre of the Czech Academy of Sciences, České Budějovice, N-1126.

Etymology: The specific name of this nematode relates to the genitive form of the generic name of the host.

Remarks. Existing taxonomic problems of the numerous species of Cucullanus due to a rather uniform morphology and often inadequate descriptions of these nematodes make a detailed comparison among all of these species impossible (Moravec et al. 2005). Therefore, these parasites are mostly dealt with according to their host groups or their zoogeographical region, as has been described by Petter (1974) and Moravec et al. (1997), respectively.

Cucullanus epinepheli $\mathrm{sp}$. n. can be easily distinguished from all its congeners by the unique structure of the an- 

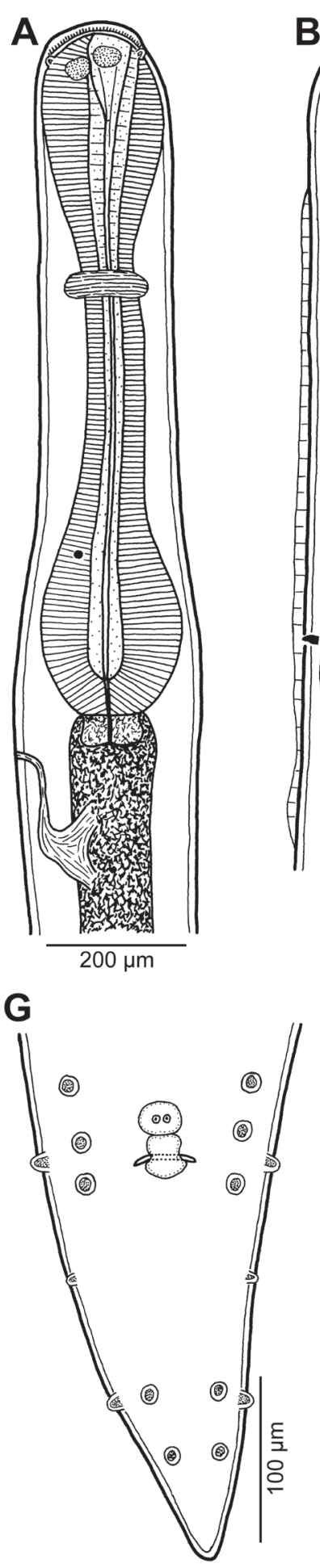
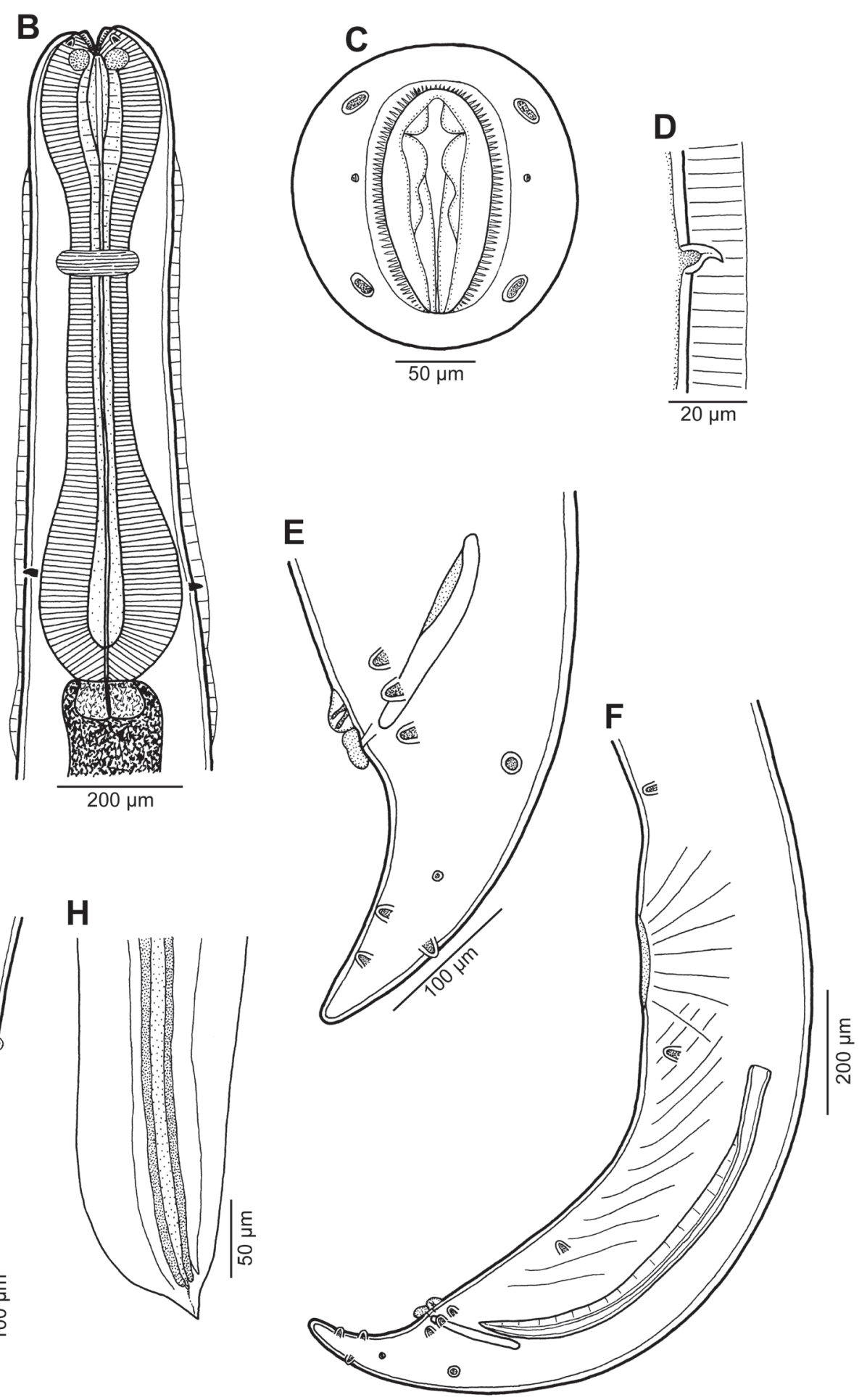

Fig. 1. Cucullanus epinepheli sp. n., male from Epinephelus chlorostigma (Valenciennes). A, B - anterior end, lateral and dorsoventral views; $\mathbf{C}$ - cephalic end, apical view; $\mathbf{D}$ - deirid and part of cervical ala, dorsoventral view; $\mathbf{E}$ - tail, lateral view; $\mathbf{F}$ - posterior end of body, lateral view; $\mathbf{G}$ - tail, ventral view; $\mathbf{H}$ - distal end of spicule.

terior cloacal lip. Moreover, of the many species of $\mathrm{Cuc}$ ullanus, the presence of cervical lateral alae, as found in C. epinepheli, was described only in Cucullanus (Truttaedacnitis) truttae Fabricius, 1794, a parasite mainly of freshwater salmonids (Salmonidae) in the Holarctic (see Moravec and Malqvist 1977, Moravec 2013). The experimental study of the larval morphogenesis of $C$. truttae showed that the third- and fourth-stage larvae of this spe- cies possessed rather wide cervical alae, whereas only narrow alae remained in conspecific adults after the last larval moult (Moravec 1979). Nevertheless, C. truttae differs considerably from $C$. epinepheli in other morphological features, such as the structure of the cephalic end, a more anterior location of deirids and the excretory pore relative to the oesophago-intestinal junction, the posterior portion of the oesophagus distinctly narrower than the pseudobuc- 


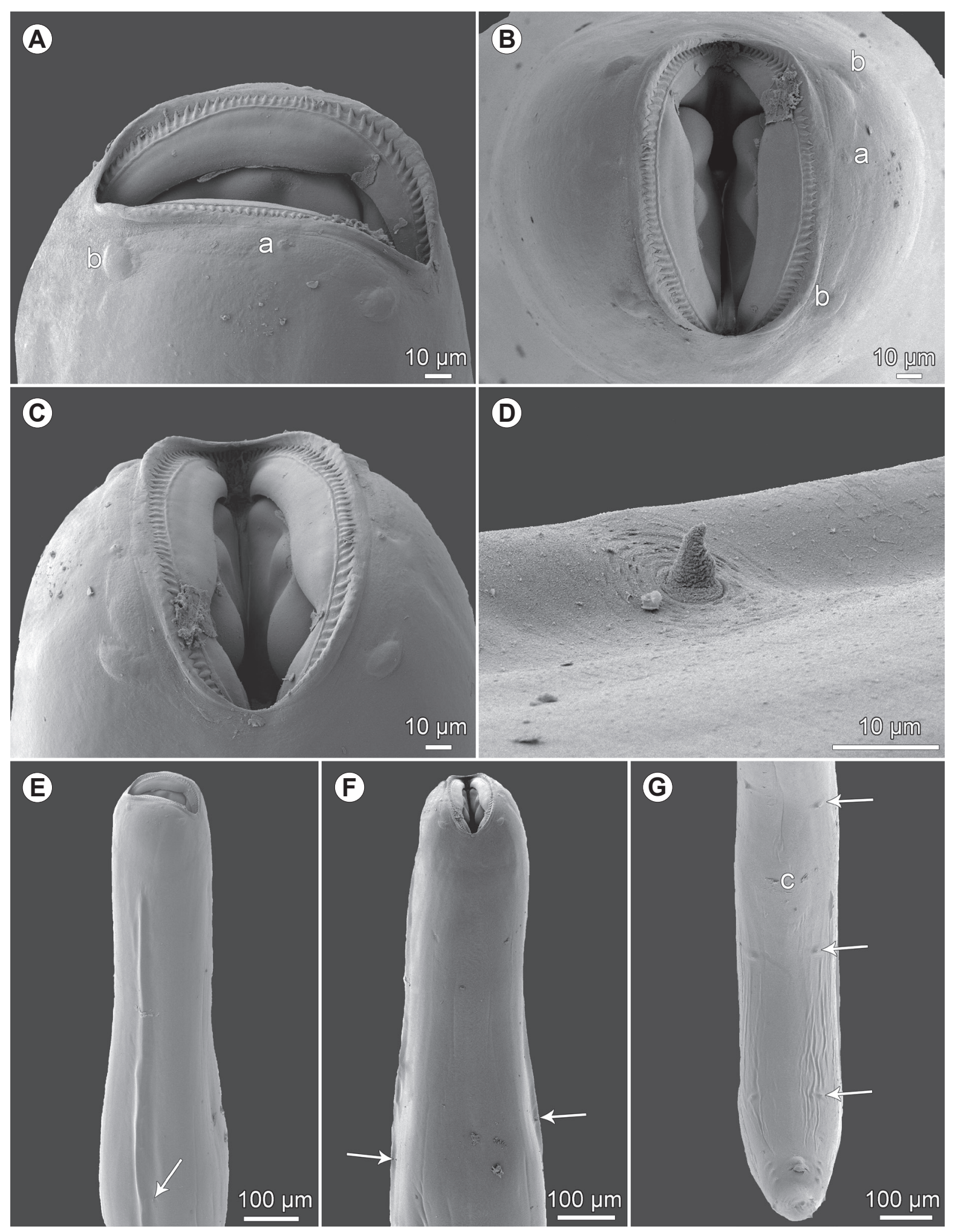

Fig. 2. Cucullanus epinepheli sp. n., scanning electron micrographs of male from Epinephelus chlorostigma (Valenciennes). A-C - cephalic end,lateral, apical and subdorsal views, respectively; D - deirid; E, F - anterior end of body with distinct cervical alae, lateral and dorsal views, respectively (arrows indicate deirids); $\mathbf{G}$ - posterior end of body, ventral view (arrows indicate papillae of first three preanal pairs. Abbreviations: a - amphid; b - cephalic papilla; c - ventral sucker.

cal capsule, and the absence of the median precloacal papilla and the markedly elevated anterior cloacal lip with a posterior outgrowth (Berland 1970, Moravec 2013).

Another feature of $C$. epinepheli is the presence of two small papillae on the median precloacal elevation. The presence of a single median precloacal papilla was found in several Cucullanus spp., e.g. C. australiensis Baylis, 1927, C. baylisi Campana-Rouget, 1961, C. bonaerensis Lanfranchi, Timi et Sardella, 2004, C. bourdini, C. costaricensis López-Caballero, Osorio-Sarabia et García-Pri- 



Fig. 3. Cucullanus epinepheli sp. n., scanning electron micrographs of male from Epinephelus chlorostigma (Valenciennes). A - posterior end of body, lateral view (arrows indicate papillae of first three preanal pairs); B - tail, lateral view (arrow indicates phasmid); C - same, ventral view; D - region of cloaca, ventral view. Abbreviations: c - ventral sucker; d - cloaca; e - precloacal median domeshaped elevation containing two small papillae; $\mathrm{f}$ - outgrowth of anterior cloacal lip covering cloacal aperture.

eto, 2009, C. extraneus Li, Ali, Zhao, Lü et Xu, 2016, C. gastrophysi Pereira, Vieira et Luque in Vieira et al., 2015, C. hainanensis Xu, Zhang et Li, 2014, C. mormyri Moravec et Scholz, 2017, C. mycteropercae Mejía-Madrid et Guillén-Hernández, 2011, C. oceaniensis Moravec, Sasal, Würtz et Taraschewski, 2005, C. pargi GonzálezSolís, Tuz-Paredes et Quintal-Loria, 2007, C. pedroi Timi et Lanfranchi, 2006, C. protrudens Pereira, Vieira et Luque in Vieira et al., 2015, C. pseudopercis Pereira, Vieira et Luque in Vieira et al., 2015, C. rastrelligeri Yooyen, Moravec et Wongsawad, 2011, C. robustus Yamaguti, 1935 and $C$. thaiensis Yooyen, Moravec et Wongsawad, 2011 (see e.g. Mejía-Madrid and Guillén-Hernández 2011, Moravec and Justine 2011a, Vieira et al. 2015, Li et al. 2016, Moravec and Scholz 2017). However, in addition to $C$. epinepheli, the median precloacal elevation with two papillae has been described only in C. genypteri Sardella, Navone et Timi, 1997 (see Vieira et al. 2015).

However, as mentioned above, the presence of the elevated anterior cloacal lip provided with a conspicuously large posterior outgrowth covering the cloacal aperture of males of $C$. epinepheli is unique among species of $\mathrm{Cucul}$ lanus described to date. The elevated anterior cloacal lip bearing an outgrowth has also been described for C. mycteropercae, but, in this case, the outgrowth is small, oriented ventrally and not covering the cloacal aperture (see Mejía-Madrid and Guillén-Hernández 2011). As revealed by SEM, a precloacal structure somewhat similar to that in C. mycteropercae is also present in C. oceaniensis from Anguilla spp. (Anguillidae) in some South Pacific islands (Moravec et al. 2005), but the small outgrowth appears to protrude from the base of the anterior cloacal lip. According to Rasheed (1968), the males of C. theraponi Rasheed, 1968 from marine fishes off Pakistan have both cloacal lips conspicuously elevated and each of them bears a distinct conical, ventrally oriented projection (however, this has not yet been confirmed by SEM); this species differs from $C$. epinepheli mainly in the absence of a ventral sucker.

To date, C. mycteropercae, a parasite of Mycteroperca bonaci Poey (Serranidae) from off the coast of Yucatán, Mexico, has been the only nominal species of Cucullanus described from a serranid fish host (Mejía-Madrid and Guillén-Hernández 2011). Accordingly, C. epinephe$l i$ is the second species known to parasitise hosts of this fish family. Both of these species are characterised by the presence of the elevated anterior cloacal lip with a distinct 
outgrowth (see above), in which they differ from all other congeners. In addition to these nominal species, Justine et al. (2010) mentioned the presence of an unidentified Cucullanus sp. in Variola louti (Forsskål) and Variola albimarginata Baissac off New Caledonia.

Cucullanus stossichi Barreto, 1922 was reported from a North American serranid fish Mycteroperca venenosa (Linnaeus), but this inadequately described species was based on data of Linton (1905, 1907) (see Barreto 1922), who had evidently misidentified his specimens, collected from hosts belonging to several families, as Heterakis foveolata (= Cucullanus foveolatus Rudolphi, 1809, a junior synonym of $C$. cirratus Müller, 1777, a parasite of gadiform fishes). However, in view of the improbability of a single cucullanid species occurring in such a wide range of hosts, it is probable that $C$. stossichi represents several species and should be considered a species inquirenda. Bharathalakshmi and Sudha (1999) reported Cucullanus fastigatus (= Dichelyne fastigatus Chandler, 1935) from Epinephelus undulosus (Quoy et Gaimard) (Serranidae) and two other host species belonging to the Haemulidae and Sciaenidae off India, but it was a probable misidentification of these nematodes (Moravec et al. 2011).

Cucullanus epinepheli is the second known nominal species of this genus reported from marine fishes in New Caledonian waters.

Although only three specimens of E. chlorostigma were collected off New Caledonia for parasitology, identifications and descriptions of new species were obtained for copepods (Justine et al. 2010), digeneans (Bray and Justine 2011, 2012, 2013), cestodes (Beveridge et al. 2014), monogeneans (Justine and Henry 2010, Schoelinck et al. 2012) and nematodes (the present paper).

Camallanidae Railliet et Henry, 1915

\section{Procamallanus (Spirocamallanus) sinespinis sp. $\mathrm{n}$}

Figs. 4, 5

ZooBank number for species:

urn:lsid:zoobank.org:act:583E0B28-A95D-4339-8A29-08D794FD9E89

Description: Medium-sized nematode with finely transversely striated cuticle. Mouth aperture oval, surrounded by 12 submedian cephalic papillae arranged in three circles, each formed by four papillae; papillae of outer circle distinctly larger; each of four small inner papillae located near margin of oral aperture accompanied by distinct proximal pore; pair of small lateral amphids present (Figs. 4D, 5A,B,D). Buccal capsule orange-brown, thickwalled, slightly longer than wide, with simple, well-developed basal ring. Maximum width/length ratio of buccal capsule $1: 1.16-1.33$. Inner surface of capsule provided with 10-12 spiral ridges in lateral view, 2-3 of them being incomplete (Fig. 4A-C). Muscular oesophagus somewhat shorter than glandular oesophagus; both parts of oesophagus slightly expanded near their posterior ends (Fig. 1A). Intestine brown, narrow. Deirids small, simple, rounded, situated at short distance posterior to base of buccal cap- sule (Figs. 4B-C,H, 5A). Excretory pore approximately at level of junction of both parts of oesophagus (Fig. 4A).

Male (three specimens; measurements of holotype in parentheses). Length of body 19.7-23.2 mm (23.2 mm), maximum width 313-340 (313). Buccal capsule including basal ring 90-96 (96) long, its width 78-81 (81); basal ring 9-15 (12) long and 57-60 (60) wide. Maximum width/ length ratio of buccal capsule $1: 1.16-1.19$ (1:1.19). Spiral ridges 10-12 (12), 2-3 (2) of which incomplete. Length of muscular oesophagus 530-585 (585), maximum width 90-105 (90); length of glandular oesophagus 734-843 (734), maximum width 102-163; length ratio of muscular and glandular oesophagus $1: 1.25-1.59$ (1: 1.25). Length of entire oesophagus and buccal capsule representing $6-7 \%(6 \%)$ of body length. Deirids, nerve ring and excretory pore 159-177 (159), 326-340 (340) and 571-653 (653) from anterior extremity, respectively.

Posterior end of body ventrally bent, provided with wide, vesiculated caudal alae supported by pedunculate papillae; anteriorly alae interconnected, forming a kind of pseudosucker, and posteriorly reaching caudal terminal knob (Figs. 4F,G. 5C,E,F). Preanal papillae: 3 pairs of subventral pedunculate papillae, of which second and third pairs closer to each other than first and second pairs; postanal papillae: 6 pairs of pedunculate papillae, 4 subventral and 2 lateral (last pair representing phasmids); additional 2 pairs of small, transversely-elongate sessile ventral papillae surrounding cloacal opening (Figs. 4F, G, 5C,E,F).

Spicules similar in shape, unequal, with sharply pointed distal ends; large (right) spicule 465-525 (465) long; small (left) spicule less well sclerotised, 218-231 (218) long. Length ratio of spicules $1: 2.13-2.27$ ( $1: 2.13)$. Gubernaculum absent. Tail conical, 150-177 (163) long, with small terminal cuticular knob (Figs. 4F,G, 5C,E,F).

Female (one ovigerous specimen, allotype). Length of body $35.7 \mathrm{~mm}$, maximum width 612 . Buccal capsule including basal ring 120 long and 90 wide; basal ring 12 long and 96 wide. Maximum width/length ratio of buccal capsule $1: 1.33$. Number of spiral ridges 12 , of which two incomplete. Length of muscular oesophagus 571, maximum width 95 ; length of glandular oesophagus 1,006, maximum width 150; length ratio of muscular and glandular oesophagus $1: 1.76$. Length of entire oesophagus and buccal capsule representing $5 \%$ of body length. Deirids, nerve ring and excretory pore 225, 408 and 789, respectively, from anterior extremity. Vulva equatorial, $17.7 \mathrm{~mm}$ from anterior extremity, at $50 \%$ of body length. Vulval lips not elevated. Uterus filled with numerous eggs. Tail broad, rounded, its posterior end abruptly narrowed to form terminal digit-like protrusion with smooth, rounded tip; length of entire tail 270; digit-like protrusion 60 long, 21 wide (Fig. 4E).

Type host: Silver grunt Pomadasys argenteus (Forsskål) (Perciformes: Haemulidae). The two fish examined ranged between $230-250 \mathrm{~mm}$ in fork length and 227-262 $\mathrm{g}$ in weight.

Site of infection: Digestive tract.

Type locality: Off New Caledonia (collected 29 April 2010).

Prevalence and intensity: 1 fish infected/2 fish examined; 4 nematodes. 

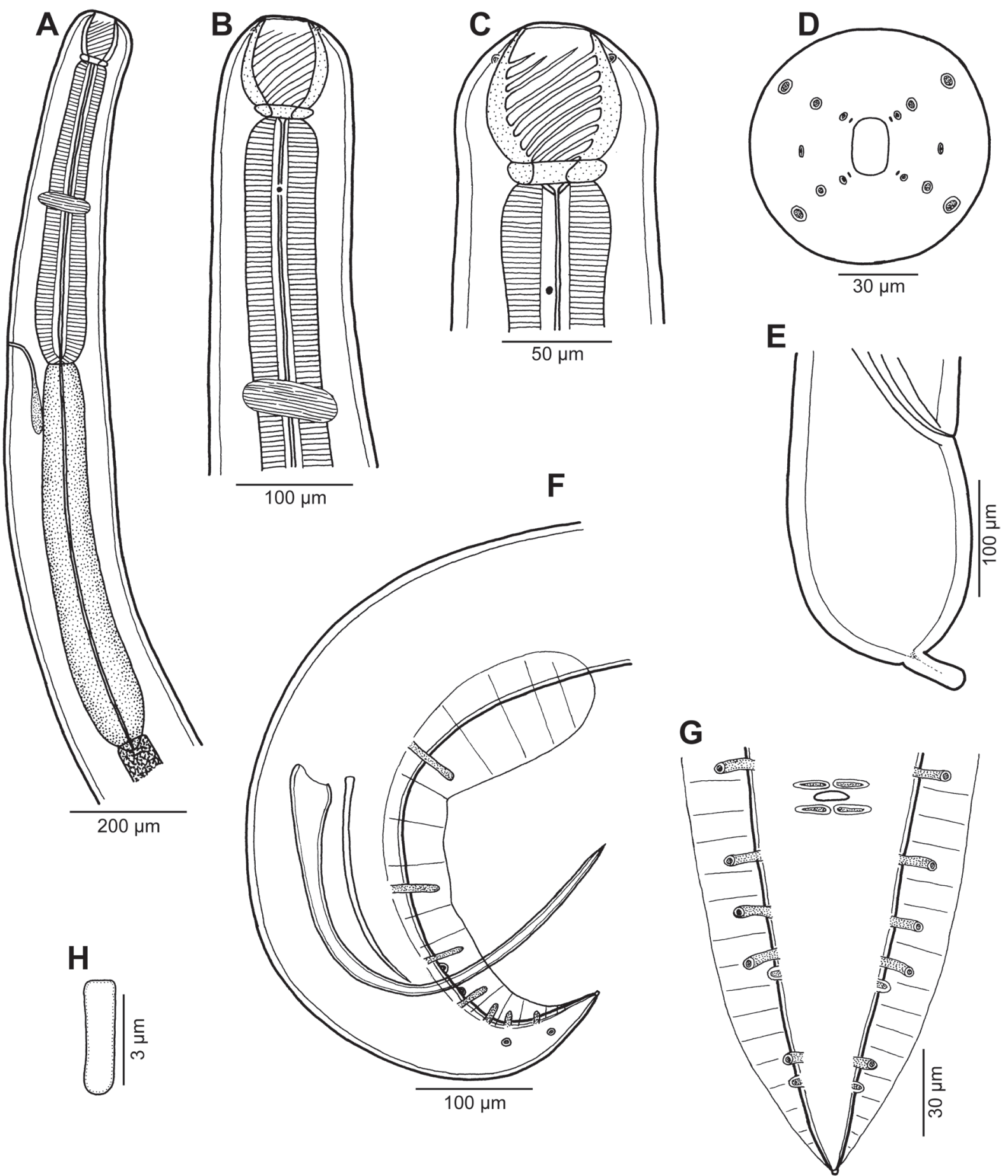

Fig. 4. Procamallanus (Spirocamallanus) sinespinis sp. n. from Pomadasys argenteus (Forsskål). A - anterior, oesophageal part of male body, lateral view; B - anterior end of subgravid female, lateral view; C, D - cephalic end of male, lateral and apical views, respectively; $\mathbf{E}$ - tail of subgravid female, lateral view; $\mathbf{F}$ - posterior end of male, lateral view; $\mathbf{G}$ - tail of male, ventral view; $\mathbf{H}$ - shape of deirid.

Deposition of type specimens: Holotype and allotype, Muséum National d'Histoire Naturelle, Paris, MNHN JNC 3156; 1 male paratype, Helminthological Collection of the Institute of Parasitology, Biology Centre of the Czech Academy of Sciences, České Budějovice, N-1127.

Etymology: The specific name of this nematode sinespinis consists of the Latin preposition sine (= without) and the ablative plural of the substantive spina (= thorn, spike), i.e. sine spinis (= without spikes); this relates to the fact that there are no cuticular spikes on the female tail tip.
Remarks. The general morphology of the new species shows that it belongs to the subgenus Spirocamallanus Olsen, 1952 of the genus Procamallanus in the conception of Moravec and Thatcher (1997). By its morphology, it should be assigned to the group of Spirocamallanus species characterised by the presence of wide caudal alae, three pairs of pedunculate preanal papillae and two unequal spicules, that are mostly parasites of marine fishes (Petter 1979). A great majority of species of this group is 


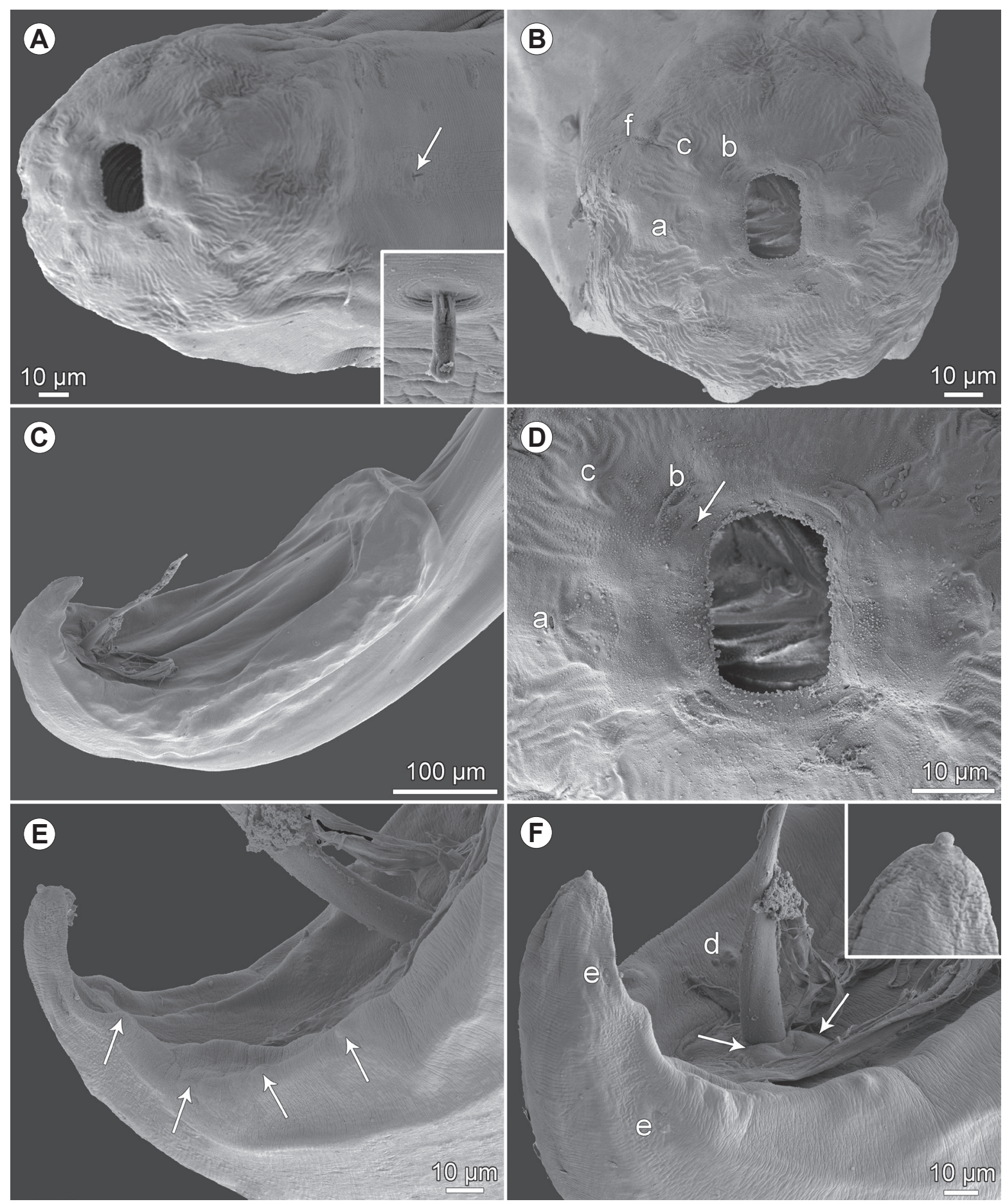

Fig. 5. Procamallanus (Spirocamallanus) sinespinis sp. n., scanning electron micrographs of male from Pomadasys argenteus (Forsskål). A - anterior end of male, sublateral view (arrow indicates deirid); insert: deirid; $\mathbf{B}$ - cephalic end, apical view; $\mathbf{C}$ - posterior end showing pseudosucker formed by caudal alae, sublateral view; D - region of oral aperture, apical view (arrow indicates proximal pore); $\mathbf{E}$ - tail, sublateral view (arrows indicate subventral postanal papillae); $\mathbf{F}$ - same, another view (arrows indicate circumcloacal papillae); insert: tail tip with terminal knob-like projection. Abbreviations: a - amphid; b - cephalic papilla of inner circle; $\mathrm{c}$ - cephalic papilla of middle circle; $\mathrm{d}$ - papilla of last preanal pair; $\mathrm{e}$ - lateral postanal papilla; $\mathrm{f}$ - cephalic papilla of outer circle.

characterised by the presence of two caudal spikes, one dorsal and one ventral, on a digitiform projection of the female tail (González-Solís et al. 2002, Yooyen et al. 2011), whereas these are lacking in only a few species. As pointed out by Petter et al. (1977), Rigby and Adamson (1997) and Moravec et al. (2006), the shape and structure of the female tail appears to be constant within species of Procamallanus (Spirocamallanus).

Based on the shape of the female tail and the absence of any terminal spikes, $P$. (S.) sinespinis sp. n. resembles only P. (S.) macaensis (Vicente et Santos, 1972) from several species of marine fishes off Brazil (Vicente and San- 
tos 1972, Luque et al. 2011), P. (S.) mexicanus Moravec, Salgado-Maldonado et Caspeta-Mandujano, 2000 from the freshwater cichlid Cichlasoma geddesi (Regan) (Cichlidae) in Mexico (Moravec et al. 2000) and P. (S.) murrayensis Johnston et Mawson, 1940 from freshwater perciform fishes Pseudaphritis urvillii (Valenciennes) (Pseudophritidae), Macquaria colonorum (Günther) and M. ambigua (Richardson) (both Percichthyidae) in Australia (Johnston and Mawson 1940).

However, in contrast to the new species, $P$. (S.) macaensis has the right spicule markedly longer (610-620 $\mu \mathrm{m}$ vs $465-525 \mu \mathrm{m})$, whereas the same spicule is distinctly shorter $(290 \mu \mathrm{m})$ in $P$. (S.) murrayensis. The right spicule of $P$. (S.) mexicanus is rather similar $(456-480 \mu \mathrm{m} v \mathrm{~s}$ 465-525 $\mu \mathrm{m})$ to that of $P$. (S.) sinespinis and both of these species have the same number (10-12) of spiral ridges in the buccal capsule, but the arrangement of their postanal papillae is different. The male tail tip of $P$. (S.) mexicanus bears a conical cuticular spike ( $v s$ a knob-like structure in $P$. (S.) sinespinis) and the female tail of the former species is shorter $(147 \mu \mathrm{m} v s 270 \mu \mathrm{m})$ and has a different shape (its anterior portion is narrow and conical $v s$ broad and posteriorly rounded). Moreover, $P$. (S.) mexicanus and $P$. (S.) murrayensis are parasites of freshwater fishes, whereas the host of $P$. $(S$.) sinespinis is the marine fish.

Moravec et al. (2006) reported a subgravid female nematode, designated as Procamallanus (Spirocamallanus) sp. 3, from the marine fish Scolopsis bilineata (Bloch) (Nemipteridae) from off New Caledonia. The shape of its tail is similar to that of $P$. ( $S$.) sinespinis, but since the number of spiral ridges in the buccal capsule is different (13 vs 10-12), as well as the location of deirids (at the level of the nerve ring $v s$ at a short distance posterior to the buccal capsule), this specimen clearly represents another species.

Procamallanus $(S$.) sinespinis is the fifth nominal species of the subgenus Spirocamallanus reported from marine fishes in New Caledonian waters.

Acknowledgements. Sophie Olivier and Charlotte Schoelinck, former JLJ's students, participated in the parasitological survey, as did Bernard Marchand. Authors' thanks are also due to the staff of the Laboratory of Electron Microscopy, Institute of Parasitology, Biology Centre of the CAS, České Budějovice for their technical assistance, and to Blanka Škoríková of the same Institute for help with the illustrations. This study was partly supported by the Czech Science Foundation (Grant No. P505/12/G112) and by institutional support (RVO: 60077344, Institute of Parasitology, BC CAS).

\section{REFERENCES}

BAILLON N. 1991: Otolithomérie en milieu tropical: application à trois espèces du lagon de Nouvelle-Calédonie (Vol. Travaux et Documents Microédités, TDM 113). Éditions de l'ORSTOM, Bondy, $290 \mathrm{pp}$.

BArreto A.L. DE B. 1922: Revisão de familia Cucullanidae Barreto, 1916. Mem. Inst. Oswaldo Cruz 14: 68-87, Plts. 33-46.

BERLAND B. 1970: On the morphology of the head in four species of the Cucullanidae (Nematoda). Sarsia 43: 15-64.

Beveridge I., Bray R.A., Cribb T.H., Justine J.-L. 2014: Diversity of trypanorhynch metacestodes in teleost fishes from coral reefs off eastern Australia and New Caledonia. Parasite 21: 60.

Bharathalakshmi B., Sudha M. 1999: Redescription of Cucullanus fastigatus (Chandler, 1935) Rasheed, 1968 from three new host fishes Pomadasys maculatus Bloch, Dendrophysa russelli Cuvier and Epinephelus undulosus Quoy and Gaimard from Visakhapatnam. Uttar Pradesh J. Zool. 19: 113-116.

Bray R.A., Justine J.-L. 2011: Acanthocolpidae (Digenea) of marine fishes off New Caledonia, with the descriptions of two new species. Folia Parasitol. 58: 35-47.

Bray R.A., Justine J.-L. 2012: A review of the Lepocreadiidae (Digenea, Lepocreadioidea) from fishes of the waters around New Caledonia. Acta Parasitol. 57: 247-272.

Bray R.A., Justine J.-L. 2013: Bucephalidae (Digenea) from epinephelines (Serranidae: Perciformes) from the waters off New Caledonia, including Neidhartia lochepintade n. sp. Parasite 20: 56.

Froese R., Pauly D. (Eds.) 2016: FishBase. World Wide Web electronic publication. www.fishbase.org, version 11/2016.

GonzÁlez-Solís D., Moravec F., Vidal-Martínez V.M. 2002: Procamallanus (Spirocamallanus) chetumalensis n. sp. (Nematoda: Camallanidae) from the Malayan sea catfish, Ariopsis assimilis, off the Caribbean coast of Mexico. J. Parasitol. 88: 765-768.

Johnston T.H., Mawson P.M. 1940: Some nematodes parasitic in Australian freshwater fish. Trans. R. Soc. S. Aust. 64: 340-352.

Justine J.-L. 2010: Parasites of coral reef fish: how much do we know? With a bibliography of fish parasites in New Caledonia. Belg. J. Zool. 140 (Suppl.): 155-190.
Justine J.-L., Beveridge I., Boxshall G.A., Bray R.A., Moravec F., Trilles J.-P., Whittington I.D. 2010: An annotated list of parasites (Isopoda, Copepoda, Monogenea, Digenea, Cestoda and Nematoda) collected in groupers (Serranidae, Epinephelinae) in New Caledonia emphasises parasite biodiversity in coral reef fish. Folia Parasitol. 57: 237-262.

Justine J.-L., BRiAnd M.J., BRAY R.A. 2012: A quick and simple method, usable in the field, for collecting parasites in suitable condition for both morphological and molecular studies. Parasitol. Res. 111: 341-351.

Justine J.-L., Henry É. 2010: Monogeneans from Epinephelus chlorostigma (Val.) (Perciformes: Serranidae) off New Caledonia, with the description of three new species of diplectanids. Syst. Parasitol. 77: 81-105.

Li L., Ali A.H., Zhao W.-T., Lü L., Xu Z. 2016: First report on nematode parasite infection in the yellowbar angelfish Pomacanthus maculosus (Perciformes: Pomacanthidae) from the Iraqi coral reef, with description of a new species of Cucullanus (Nematoda: Ascaridida) using the integrated approaches. Parasitol. Int. 65: 677-684.

Linton E. 1905: Parasites of fishes of Beaufort, North Carolina. Bull. U. S. Bureau Fish. 24: 321-428.

Linton E. 1907: Notes on parasites of Bermuda fishes. Proc. U. S. Nat. Mus. 33: 85-126.

Luque J.L., Aguiar J.C., Vieira F.M., Gibson D.I., Santos C.P. 2011: Checklist of Nematoda associated with the fishes of Brazil. Zootaxa 3082: 1-88.

Mejía-Madrid H.H., Guillén-Hernández S. 2011: A new cucullanid from the black grouper Mycteroperca bonaci (Osteichthyes: Serranidae) off the coast of Yucatán, Mexico. J. Parasitol. 97: $122-127$.

Moravec F. 1979: Observations on the development of Cucullanus (Truttaedacnitis) truttae (Fabricius, 1794) (Nematoda: Cucullanidae). Folia Parasitol. 26: 295-307.

Moravec F. 2013: Parasitic Nematodes of Freshwater Fishes of Europe. Revised second edition. Academia, Prague, 601 pp.

Moravec F., Justine J.-L. 2011a: Cucullanid nematodes (Nematoda: Cucullanidae) from deep-sea marine fishes off New Cal- 
edonia, including Dichelyne etelidis n. sp. Syst. Parasitol. 78: 95-108.

Moravec F., Justine J.-L. 2011b: New data on the morphology of Procamallanus (Procamallanus) annulatus and Procamallanus (Spirocamallanus) monotaxis (Nematoda: Camallanidae) from marine fishes off New Caledonia. Helminthologia 48: 41-50.

Moravec F., Justine J.-L. 2016: Nematode parasites of four species of Carangoides (Osteichthyes: Carangidae) in New Caledonian waters, with a description of Philometra dispar $\mathrm{n}$. $\mathrm{sp}$. (Philometridae). Parasite 23: 40.

Moravec F., Justine J.-L., Rigby M.C. 2006: Some camallanid nematodes from marine perciform fishes off New Caledonia. Folia Parasitol. 53: 223-239.

Moravec F., Kohn A., Fernandes B.M.M. 1997: New observations on seuratoid nematodes parasitic in fishes of the Paraná River, Brazil. Folia Parasitol. 44: 209-223.

Moravec F., Levron C., De Buron I. 2011: Morphology and taxonomic status of two little-known nematode species parasitizing North American fishes. J. Parasitol. 97: 297-304.

Moravec F., Malqvist B. 1977: Records of Cucullanus truttae (Fabricius, 1794) (Nematoda: Cucullanidae) from Swedish brook lampreys, Lampetra planeri (Bloch). Folia Parasitol. 24 323-329.

Moravec F., Salgado-Maldonado G., Caspeta-Mandujano J. 2000: Three new Procamallanus (Spirocamallanus) species from freshwater fishes in Mexico. J. Parasitol. 86: 119-127.

Moravec F., Sasal P., Würtz J., Taraschewski H. 2005: $\mathrm{Cu}$ cullanus oceaniensis sp. n. (Nematoda: Cucullanidae) from eels (Anguilla spp.). Folia Parasitol. 52: 343-348.

Moravec F., Scholz T. 2017: Some nematodes, including two new species, from freshwater fishes in the Sudan and Ethiopia. Folia Parasitol. 64: 010.

Moravec F., Thatcher V.E. 1997: Procamallanus (Denticamallanus subgen. n.) dentatus n. sp. (Nematoda: Camallanidae) from the characid fish, Bryconops alburnoides, in the Brazilian Amazon. Parasite 4: 239-243.
Petter A.-J. 1974: Essai de classification de la famille des Cucullanidae. Bull. Mus. Nat. Hist. Nat., Paris, $3^{\mathrm{e}}$ sér., No. 255, Zoologie 177: 1469-1490.

Petter A.-J. 1979: Essai de classification de la sous-famille des Procamallaninae (Nematoda, Camallanidae). Bull. Mus. Nat. Hist. Nat., Paris, Sect. A, Zoologie 1: 991-1008.

Petter A.-J., Golvan Y.J., Tcheprakoff R. 1977: Nématodes de poissons de rivière en Guadeloupe. Bull. Mus. Nat. Hist. Nat., Paris, $3^{\text {e }}$ sér., No. 428, Sect. A, Zoologie 298: 159-171.

Petter A.-J., Le Bel J. 1992: Two new species in the genus $\mathrm{Cu}$ cullanus (Nematoda-Cucullanidae) from the Australian region. Mem. Inst. Oswaldo Cruz 87 (Suppl. I): 201-206.

RasheEd S. 1968: The nematodes of the genus Cucullanus Mueller, 1777, from the marine fish of Karachi coast. An. Esc. Nac. Cienc. Biol., México, 15: 23-59.

Rigby M.C., Adamson M.L. 1997: Spirocamallanus species of French Polynesian coral reef fishes. Can. J. Zool. 75: 1270-1279.

Schoelinck C., Cruaud C., Justine J.-L. 2012: Are all species of Pseudorhabdosynochus strictly host specific? - A molecular study. Parasitol. Int. 61: 356-359.

Schoelinck C., Hinsinger D.D., Dettaï A., Cruaud C., JusTINE J.-L. 2014: A phylogenetic re-analysis of groupers with applications for ciguatera fish poisoning. PLoS ONE 9: e98198.

Vicente J.J., Santos E. 1972: Sobre um novo nematódeo camalanídeo parasito de peixe marinho (Nematoda, Camallanoidea). Atas Soc. Biol. Rio de Janeiro 15: 145-147.

Vieira F.M., Pereira F.B., Pantoja C., Soares I.A., Pereira A.N., Timi J.T., Scholz T., LuQue J.L. 2015: A survey of nematodes of the genus Cucullanus Müller, 1777 (Nematoda, Seuratoidea) parasitic in marine fishes off Brazil, including description of three new species. Zootaxa 4039: 289-311.

Yooyen T., Moravec F., Wongsawad C. 2011: Two new sibling species of Procamallanus (Spirocamallanus) (Nematoda: Camallanidae) from marine fishes in the Gulf of Thailand. J. Parasitol. 97: 931-938.

Cite this article as: Morave F., Justine J.-L.. 2017: Two new species of nematode parasites, Cucullanus epinepheli sp. n. (Cucullanidae) and Procamallanus (Spirocamallanus) sinespinis sp. n. (Camallanidae), from marine serranid and haemulid fishes off New Caledonia. Folia Parasitol. 64: 011. 